\title{
Exploration of selector characteristic based on electron tunneling for RRAM array application
}

\author{
Bing Song, Qingjiang Li, Husheng Liu, and Haijun Liu ${ }^{\text {a) }}$ \\ College of Electronic Science and Engineering, National University of Defense \\ Technology, Changsha 410073, China \\ a)liuhaijun@nudt.edu.cn
}

\begin{abstract}
Selector is indispensable to suppress leakage current for crossbar array of resistive random access memory. According to the nonlinear requirement, electron tunneling mechanism is firstly attempted. However, earlier studies discovered drawbacks of insufficient current density. This work aims at exploring the idealized characteristic of selector based on FowlerNordheim tunneling mechanism by selecting various materials and structures. Thereinto, current density transforms to drive voltage according to corresponding current density standard. Simulation results indicate that metal/insulator barrier and insulator thickness play key roles in determining drive voltage and nonlinearity of the tunneling selectors. Specifically, metal/insulator barrier influence drive voltage and nonlinearity, while insulator thickness mainly influence drive voltage. Thus it can help comprehend restrictions of tunneling mechanism and attempt other improvement directions.
\end{abstract}

Keywords: selector, RRAM, nonlinearity, F-N tunneling Classification: Integrated circuits

\section{References}

[1] A. Chen: "Nonlinearity and asymmetry for device selection in cross-bar memory arrays," IEEE Trans. Electron Devices 62 (2015) 2857 (DOI: 10.1109/ TED.2015.2450712).

[2] S. Lee, et al.: "Comprehensive methodology for ReRAM and selector design guideline of cross-point array," IEEE International Memory Workshop (IMW) (2015) 1 (DOI: 10.1109/IMW.2015.7150280).

[3] L. Zhang, et al:: "One-Selector one-resistor cross-point array with threshold switching selector,” IEEE Trans. Electron Devices 62 (2015) 3250 (DOI: 10. 1109/TED.2015.2461656).

[4] L. Zhang, et al.: "Ultrathin metal/amorphous-silicon/metal diode for bipolar RRAM selector applications,” IEEE Electron Device Lett. 35 (2014) 199 (DOI: 10.1109/LED.2013.2293591).

[5] L. Zhang, et al:: "High-drive current $\left(>1 \mathrm{MA} / \mathrm{cm}^{2}\right)$ and highly nonlinear $\left(>10^{3}\right) \quad$ TiN/amorphous-Silicon/TiN scalable bidirectional selector with excellent reliability and its variability impact on the 1 S1R array performance," 
IEEE International Electron Devices Meeting (IEDM) (2014) 6.8.1 (DOI: 10. 1109/IEDM.2014.7047000).

[6] M. Wang, et al:: "Conduction mechanism of a TaOx-based selector and its application in crossbar memory arrays," Nanoscale 7 (2015) 4964 (DOI: 10. 1039/C4NR06922F).

[7] B. J. Choi, et al: "Trilayer tunnel selectors for memristor memory cells," Adv. Mater. 28 (2016) 356 (DOI: 10.1002/adma.201503604).

[8] B. Govoreanu, et al:: "High-performance metal-insulator-metal tunnel diode selectors," IEEE Electron Device Lett. 35 (2014) 63 (DOI: 10.1109/LED.2013. 2291911).

[9] J. Woo, et al:: "Bidirectional selection device characteristics of ultra-thin $(<3 \mathrm{~nm}) \mathrm{TiO} 2$ layer for 3D vertically stackable ReRAM application," IEEE Silicon Nanoelectronics Workshop (SNW) (2012) 1 (DOI: 10.1109/SNW.2012. 6243332).

[10] F.-C. Chiu: "A review on conduction mechanisms in dielectric films," Adv. Mater. Sci. Eng. 2014 (2014) 578168 (DOI: 10.1155/2014/578168).

[11] M. Lenzlinger and E. H. Snow: "Fowler-Nordheim tunneling into thermally grown $\mathrm{SiO}_{2}$," J. Appl. Phys. 40 (1969) 278 (DOI: 10.1063/1.1657043).

[12] E. M. Vogel, et al.: "Modeled tunnel currents for high dielectric constant dielectrics," IEEE Trans. Electron Devices 45 (1998) 1350 (DOI: 10.1109/ 16.678572).

[13] B. Govoreanu, et al.: "Selectors for high density crosspoint memory arrays: Design considerations, device implementations and some challenges ahead," International Conference on IC Design \& Technology (ICICDT) (2015) 1 (DOI: 10.1109/ICICDT.2015.7165872).

[14] L. Zhang, et al.: "Selector design considerations and requirements for 1S1R RRAM crossbar array,” IEEE International Memory Workshop (IMW) (2014) 1 (DOI: 10.1109/IMW.2014.6849358).

[15] L. Zhang, et al.: "Cell variability impact on the one-selector one-resister crosspoint array performance," IEEE Trans. Electron Devices 62 (2015) 3490 (DOI: 10.1109/TED.2015.2439958).

[16] H. C. Wen, et al.: "Systematic investigation of amorphous transition-metalsilicon-nitride electrodes for metal gate CMOS applications," IEEE VLSI Technology Dig. Tech. Papers (2005) 46 (DOI: 10.1109/.2005.1469206).

[17] C. B. Lai and Y. J. Lee: "Leakage current mechanism of metal- $\mathrm{Ta}_{2} \mathrm{O}_{5}$-metal capacitors for memory device applications," J. Electrochem. Soc. 146 (1999) 266 (DOI: 10.1149/1.1391597).

[18] H. C. Lai, et al.: "Very low voltage $\mathrm{SiO}_{2} / \mathrm{HfON} / \mathrm{HfAlO} / \mathrm{TaN}$ memory with fast speed and good retention," Symposium on VLSI Technology Digest of Technical Papers (2016) 1 (DOI: 10.1109/VLSIT.2006.1705208).

[19] T. Matsushima and C. Adachi: "Temperature-independent electron tunneling injection in tris (8-hydroxyquinoline) aluminum thin film from high-workfunction gold electrode," Thin Solid Films 516 (2008) 5069 (DOI: 10.1016/ j.tsf.2008.02.012).

[20] S. Anandan, et al:: "Room temperature growth of $\mathrm{CuO}$ nanorod arrays on copper and their application as cathode in dye-sensitized solar cells," Mater. Chem. Phys. 93 (2005) 35 (DOI: 10.1016/j.matchemphys.2005.02.002).

[21] B. C. Lai and J. Y. Lee: "Leakage current mechanisms of metal- $\mathrm{Ta}_{2} \mathrm{O}_{5}$-metal capacitors for memory device applications," J. Electrochem. Soc. 146 (1999) 266 (DOI: 10.1149/1.1391597).

[22] S. Clima, et al.: "Determination of ultimate leakage through rutile $\mathrm{TiO}_{2}$ and tetragonal $\mathrm{ZrO}_{2}$ from $\mathrm{Ab}$ Initio complex band calculations," IEEE Electron Device Lett. 34 (2013) 402 (DOI: 10.1109/LED.2013.2238885). 
[23] A. Bouazra, et al.: "Current tunnelling in MOS devices with $\mathrm{Al}_{2} \mathrm{O}_{3} / \mathrm{SiO}_{2}$ gate dielectric,” Res. Lett. Phys. 2008 (2008) 286546 (DOI: 10.1155/2008/286546).

\section{Introduction}

Resistive random access memory (RRAM) is considered as one of the most promising candidates for next generation nonvolatile memory. It possesses the potential for high-density storage benefiting from its high scalability, fast switching speed and low power consumption [1]. The RRAM element based on crossbar array can attain feature size of $4 \mathrm{~F}^{2}$ ( $\mathrm{F}$ is the line width) or even less when multilevel is realized [2]. Nonetheless, as the array is constructed by pure RRAM element leakage current is inevitable. The leakage current not only consumes much more power that leads to the loss of RRAM advantage, but may also lead to false operation. Thus the huge-scale application of the RRAM storage is seriously impeded. As illustrated in Fig. 1, to suppress leakage current, a nonlinear and bipolar independent device called selector is integrated to the RRAM element, forming the structure called as 1S1R (1-Selector-1-RRAM) [3].

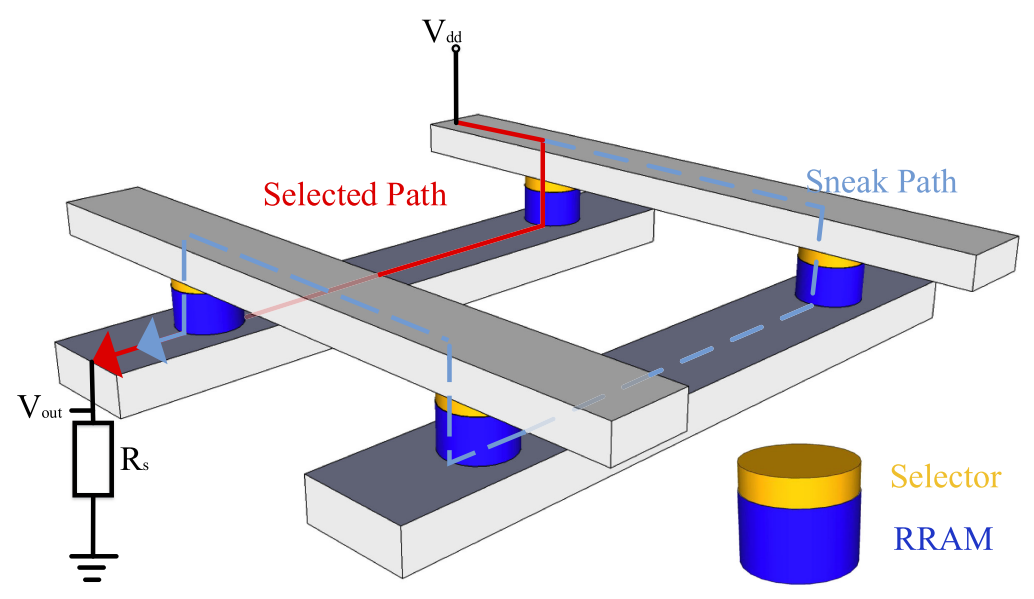

Fig. 1. Schematic of sneak path current in crossbar array

Selector is highly resistive under lower bias while highly conductive under larger bias. As a result, nonlinearity is one of the most significant characteristic. Meanwhile, compared to other mechanisms, tunneling is extensively applied and thoroughly studied semiconductor industry. Thus, selector based on tunneling mechanism is firstly realized because it embodies nonlinear current-voltage relationship with simple metal-insulator-metal structure. Early publishes demonstrated nonlinearity, fast switching speed and excellent endurance property of single selector element $[4,5,6,7,8,9]$. Furthermore, they realized leakage current suppression in single one-selector-one-RRAM (1S1R) cell [4, 5, 6, 7]. However, it is not sufficient only considering nonlinearity without sufficient drive current. When considering the current drive requirements, a customary target set forward is $1 \mathrm{MA} / \mathrm{cm}^{2}$. This value is given by the requirement of a selector to deliver a 
ability of a particular selector structure translates into lack of scalability toward $10 \mathrm{~nm}$ at the assumed $1 \mu \mathrm{A}$ operating current. Alternatively, it can translate into only being able to operate the RRAM element at less than $1 \mu \mathrm{A}$, for a $10 \mathrm{~nm}$-size cell [8]. Secondly, corresponding to first point, the voltage which current density of $1 \mathrm{MA} / \mathrm{cm}^{2}$ requires cannot be much higher than operating voltage of RRAM element. It will be tolerable in single $1 \mathrm{~S} 1 \mathrm{R}$ cell to verify function while it may lead to voltage incompatibility and operation failure in crossbar array [9]. Finally, nonlinearity is important characteristic influencing array size and power consumption. Thus higher nonlinearity with sufficient drive current means a ideal selector.

This work aims at exploring selector characteristic of F-N tunneling selector by comparing various material and structure parameters. Then it can be guideline for choosing suitable material and structure for selector devices. Meanwhile, it will also help understand restrictions of this mechanism and attempt other improvement directions. The work is organized as follows. The simulation theory and settings are presented in Section 2. The impacts of applied voltage and device parameters on current density and nonlinearity are analyzed in Section 3. The conclusion is given in Section 4.

\section{Basis theory for simulation}

Selector based on electron tunneling mechanism employs simple metal-insulatormetal structure. Tunneling theory is intensively studied, which provides convenience for simulation before fabricating real device to choose ideal material and structure. Under the circumstance of thin insulator film less than $10 \mathrm{~nm}$ or even thinner in MIM structures, tunneling can dominate the current. There exist kinds of electron tunneling mechanisms, such as F-N tunneling, direct tunneling, trapassisted tunneling, and so on [10]. Here we only consider the F-N tunneling. On one hand, F-N tunneling can occur when insulator thickness is less than $10 \mathrm{~nm}$, while direct tunneling only occurs when insulator thickness is less than $3 \mathrm{~nm}$. On the other hand, trap-assisted tunneling dominates only when there are plenty of traps in the films and the trap quantity can be reduced by implementing other process such as ALD or annealing in vacuum. Meanwhile, if there are plenty of traps in insulator such as $\mathrm{HfO}_{2}$, it easily shows nonvolatile memory property which is not included in scope. Most importantly, according to previous publishing $[4,5,6,7,8,9]$, F-N tunneling is the most prevalent underlying mechanism. As a result, simulation performs on the basis of F-N tunneling mechanism. The F-N tunneling formula is as follows [11],

$$
J_{F N}=\frac{q^{3}}{16 \pi^{2} \hbar \varphi_{b}} F_{i n}^{2} \exp \left[-\frac{4}{3} \frac{\sqrt{2 m^{*}} \varphi_{b}^{3 / 2}}{\hbar q} \frac{1}{F_{i n}}\right]
$$

Where $\mathrm{J}_{\mathrm{FN}}$ denotes current density of F-N tunneling, q denotes electron charge, $\hbar$ denotes reduced Plank's constant, $\mathrm{m}^{*}$ denotes effective mass of insulator electron, $\varphi_{\mathrm{b}}$ denotes barrier height on the interface between metal and insulator, and $F_{\text {in }}$ denotes electric field intensity. Herein $\mathrm{m}^{*}$ of the same material is constant regardless of the impact of thickness. Since 1998, Eric M. Vogel has verified the validity of this approximation method [12]. 
In the meantime, selector nonlinearity is a gauge of the selector ability to evaluate the current limiting ability and array size. Half-bias nonlinearity is defined as the ratio between the current at the applied voltage (i.e. $V_{a p p}$, at which the selector reaches it maximum drive current required to write the resistive memory element) and the current when the selector is biased at $1 / 2 V_{a p p}$, which is expressed as [13]:

$$
N L_{1 / 2}=J\left(\mathrm{~V}=\mathrm{V}_{\text {app }}\right) / J\left(\mathrm{~V}=\mathrm{V}_{\text {app }} / 2\right)
$$

Where $\mathrm{NL}_{1 / 2}$ denotes nonlinearity of half bias scheme, $J$ denotes current density. Zhang et al. investigates the selector characteristics on the overall 1S1R cell performance and extracted selector requirements using parameterized characteristics for a 1 Mbit array, taking into account the read/write margins and power consumption constraints [14]. The read margin is strongly affected by the selector nonlinearity while write margin strongly depends on the selector $V_{a p p}$. If the switching current of RRAM element is $20 \mathrm{uA}$, a selector with nonlinearity of about 4000 is required to meet all the constraints [14]. However, when it comes to the selector variability and RRAM element variability, the requirements of half-bias nonlinearity increases to 8000 [15].

Table I. Work function of different metal materials

\begin{tabular}{l|c|c|c|c|c}
\hline Electrode & TiN [16] & $\mathrm{W}[17]$ & $\mathrm{TaN}[18]$ & $\mathrm{Au}[19]$ & $\mathrm{Pt}[20]$ \\
\hline Work function $(\mathrm{eV})$ & 4.45 & 4.55 & 4.7 & 5.2 & 5.3 \\
\hline
\end{tabular}

Table II. Relative parameters of different dielectric materials

\begin{tabular}{l|l|l}
\hline Insulator & Electron affinity $(\mathrm{eV})$ & Effective electron mass \\
\hline $\mathrm{Ta}_{2} \mathrm{O}_{5}[21]$ & 3.2 & 0.3 \\
\hline $\mathrm{TiO}_{2}[22]$ & 2.95 & 0.3 \\
\hline $\mathrm{HfO}_{2}[23]$ & 2.65 & 0.17 \\
\hline $\mathrm{Al}_{2} \mathrm{O}_{3}[23]$ & 1.35 & 0.35 \\
\hline
\end{tabular}

Detailed material parameters of selected metal and insulator are depicted in Table I and Table II respectively. They are all common and widely used material in RRAM field. Metal from Table I as electrode and insulator from Table II forms the MIM structure with insulator thickness varying from 3 to $10 \mathrm{~nm}$.

\section{Results and discussion}

\subsection{Relationship between selector characteristics and applied voltage}

Firstly, the relationship between selector characteristic and applied voltage is studied. Take $\mathrm{Pt} / \mathrm{TiO}_{2} / \mathrm{Pt}$ as example, which is studied by Woo et al. and they verified the underlying mechanism is F-N tunneling [9]. Simulation results basically fitted with experiment results. As illustrated in Fig. 2, current density increase while nonlinearity decrease with increasing voltage regardless of insulator thickness. On one hand, increasing voltage bends energy band and enhances tunneling 
probability, leading to increasing current density. On the other hand, tunneling probability of half voltage and voltage are not changing in the same value. When the current density is awfully low, lower voltage increase will improve current density obviously. As a result, nonlinearity decreases when voltage increases. However, published papers usually emphasize the nonlinearity while they neglected the current density. As a consequence, there exists contradiction between current density and nonlinearity.
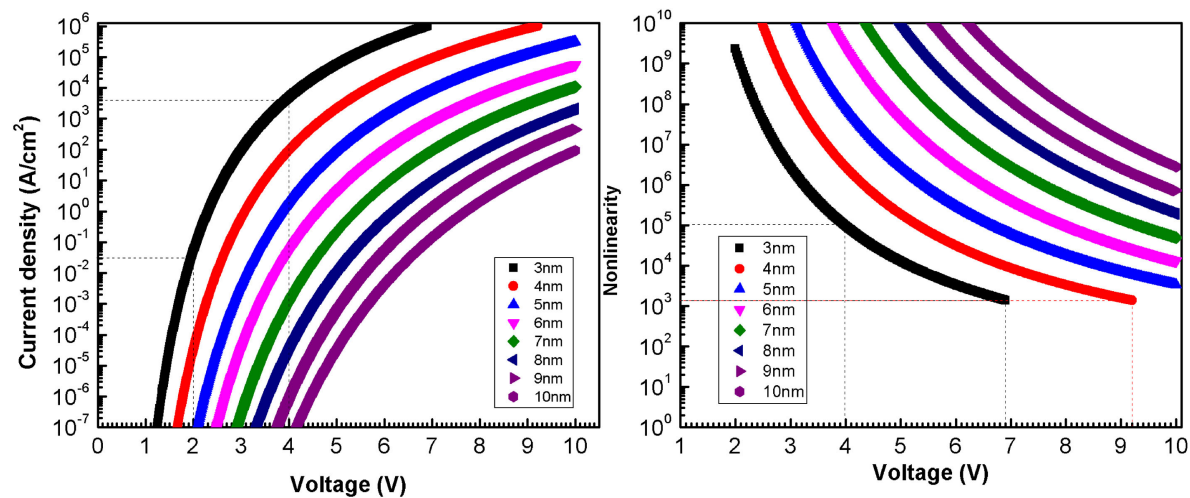

Fig. 2. Relationship between selector characteristics and applied voltages

\subsection{Relationship between selector characteristics and device pa- rameters}

Fig. 3 demonstrates the relationship between selector characteristics and device parameters-insulator thickness and barrier height. Voltage is set to $4 \mathrm{~V}$. Insulator is TiO2, and different electrode leads to various barrier height. According to the results, current density decreases with barrier height and insulator thickness. Because increasing barrier height and insulator thickness will reduce tunneling probability and current density. While nonlinearity increases with insulator thickness and barrier height. These relationships are all determined by F-N tunneling intrinsic properties.
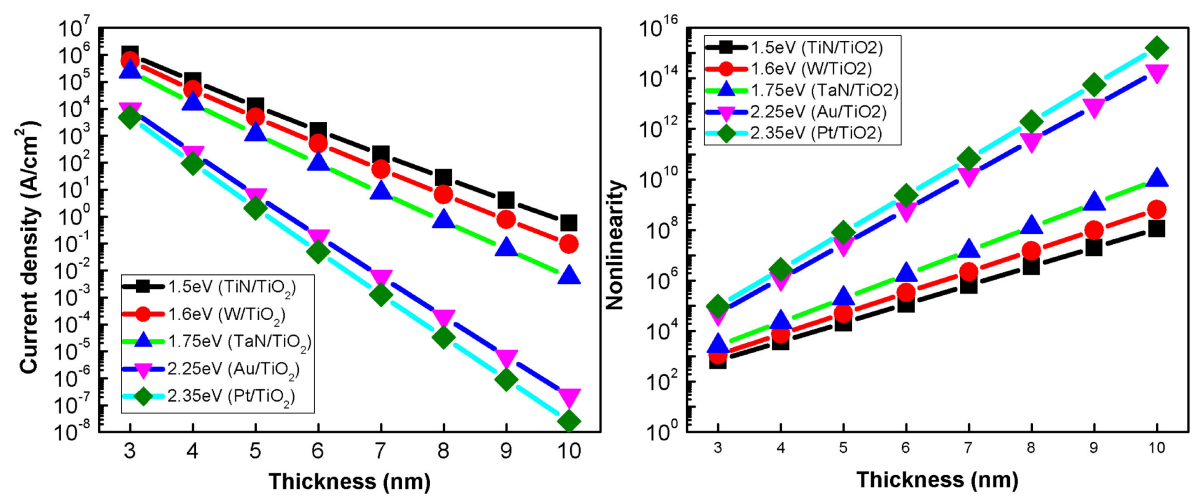

Fig. 3. Relationship between selector characteristics and device parameters 


\subsection{Exploration of idealized selector characterization based on F-N tunneling}

To explore idealized selector characterization, current density of $10^{6} \mathrm{~A} / \mathrm{cm}^{2}$ is set as the standard. Because current density is firstly guaranteed, then nonlinearity is considered as meaningful. According to above study, barrier height determined by
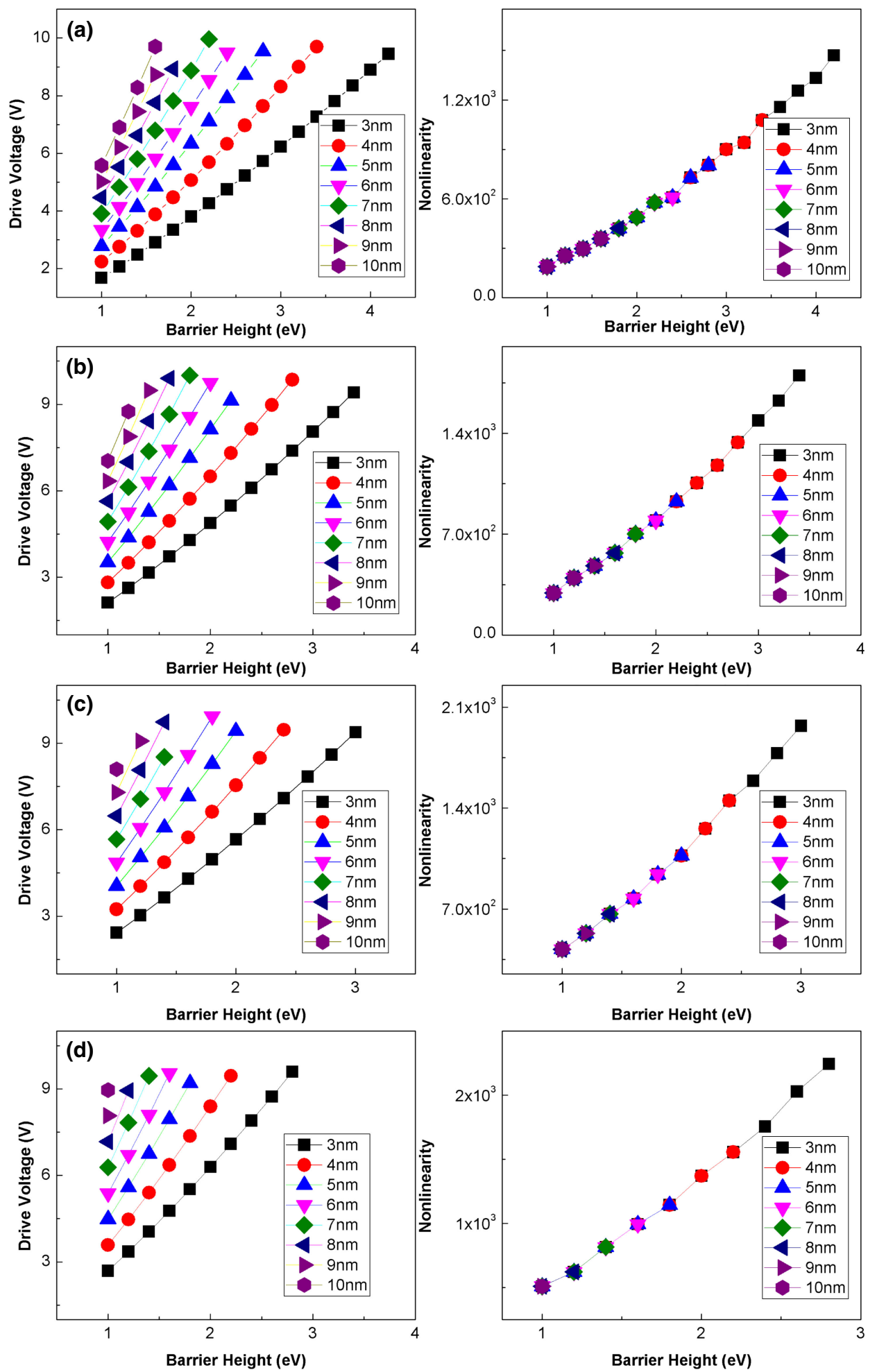
material and insulator thickness deeply impacts the characterization. Thus drive voltage for sufficient current density and corresponding nonlinearity are studied to choose appropriate material and structure. In the meantime, effective mass of insulator in tunneling mechanism is variable among various insulator materials. Hence, according to Table II, only four set of effective mass are chosen and the study results are also suitable for other insulator thickness.

As illustrated in Fig. 4, to meet the requirement of $10^{6} \mathrm{~A} / \mathrm{cm}^{2}$ current density, desired drive voltage increase with barrier height and insulator thickness. This is owing to the negative correlation between tunneling probability and barrier height and insulator thickness. In order to increase the probability, larger voltage is needed to severely distort the energy band. Meanwhile, corresponding nonlinearity is not variable to insulator thickness while increases with increasing barrier height. In addition, maximum nonlinearity is about $\sim 10^{3}$ which is not enough for highdensity arrays.

In summary, drive voltage and nonlinearity both positively correlate to the barrier height. In addition, drive voltage positively correlate to insulator thickness as well, while nonlinearity is independent of insulator thickness. As a result, in order to guarantee the drive voltage and nonlinearity, there exists a conflict. According to above results, when we design selectors we should take the tradeoff into consideration.

\section{Conclusion}

In this work, it is proved material and structural parameters can have influence on selector performance through numerical calculation. Barrier height between metal and insulator affects drive voltage and nonlinearity while insulator thickness mainly impacts drive voltage. Actually, this work aims at posing a guideline for choosing material and thickness to fabricate MIM selectors satisfying requirements. There exists tradeoff between high nonlinearity and low drive voltage for MIM selectors based on electron tunneling mechanism. It is suggested to choose materials and structural parameters in consideration of the most important requirement. In the meantime, the proposed method can be used to choose suitable parameters for experiments, which is economical and convenient of numeric simulation in advance of fabrication procedure.

\section{Acknowledgments}

This work was supported by National Natural Science Foundation of China (Grant No. 61471377 and 61604177) and NUDT Science Support Program (JC-15-04-02). 